\title{
OSTEOMYELITIS IN TURKEYS CAUSED BY YERSINIA PSEUDOTUBERCULOSIS
}

\author{
D. R. Wise AND P. K. UpPaL \\ School of Veterinary Medicine, Madingley Road, Cambridge, and \\ I.V.R.I., Mukteswar, Kumaon, U.P., India
}

SyNOVITIS and tenosynovitis are recognised as relatively common diseases of poultry, but references to osteomyelitis and arthritis are rare in the literature. Carnaghan (1966) described a spondylitis of chickens caused by Staphylococcus pyogenes. One of us (D. R. W.) has observed vertebral staphylococcal osteomyelitis in chickens and in turkeys. Nairn (1969) reported that 85 per cent. of 700 lame turkeys between 8 and 20 wk of age submitted to the University of Minnesota Veterinary Diagnostic Laboratory over a 3-yr period were suffering from osteomyelitis. He concluded that osteomyelitis was the most important known cause of locomotor difficulty in turkeys of this age in the United States, and was usually due to $S$. aureus or Escherichia coli. We have occasionally encountered staphylococcal osteomyelitis of the leg bones of turkeys, chickens and pheasants in England, but did not regard this as a major cause of lameness in poultry.

Snoeyenbos (1965) reviewed the subject of pseudotuberculosis in birds in the United States and Europe. The only published report of pseudotuberculosis in turkeys in Britain was made by Blaxland (1947). All previous reports have described death as the usual end-result of infection. Death was either sudden from septicaemia, or occurred after an illness of several weeks. The chronic cases showed post-mortem evidence of small, whitish granulomatous lesions in the viscera, particularly in the livers and spleens. Lameness or stiffness of gait has previously been described as one of the clinical signs of pseudotuberculosis, but no previous communication has reported lameness as the sole clinical sign of infection, nor have lesions of osteomyelitis associated with Yersinia pseudotuberculosis infection been described previously.

In the summer of 1970, a high incidence of lameness of sudden onset was encountered in a flock of turkey stags in England. Post-mortem examination of the affected birds revealed osteomyelitis at the ends of long bones and from these lesions an organism subsequently identified as $Y$.pseudotuberculosis was isolated. The flock had no abnormal mortality before the development of clinical signs of lameness, and there was no such mortality subsequently.

\section{Clinical AND EXPERIMENTAL FINDINGS}

Over a 2-wk period, approximately 25 per cent. of a flock of five hundred 12 -wk-old broad-breasted stag turkeys developed clinical signs of severe lameness. Growth rates were decreased, but there was no mortality. All obviously lame turkeys, numbering 125 , were slaughtered at 15 wk of age. As lameness continued to develop in considerable numbers of the surviving birds, all the remaining turkeys were slaughtered at $19 \mathrm{wk}$ of age.

Sixteen 13-wk-old lame turkeys and six clinically normal flockmates were submitted for necropsy. The viscera of all the birds appeared macroscopically normal. Routine attempts at bacterial isolation were made on horse blood agar plates from the heart-blood and livers of all 22 turkeys. No bacteria were recovered.

The long bones and joints of both left and right legs of all birds subjected to necropsy were examined. No lesions were found in any of the clinically normal turkeys. There were, however, bone lesions in at least one and usually in both legs of all the 16 lame birds, sited at the ends of the long bones in or just below the region of the growth cartilages. The lesions

Received 12 Dec. 1970; accepted 16 June 1971.

J. MED. MICROBIOL. - VOL. 5 (1972) 
had yellowish, caseo-necrotic centres surrounded by zones of clear gelatinous material. In a few cases the joint cavities adjacent to the bone lesions showed a grey mucoid exudate. No bacteria were isolated from the joint exudate on horse blood agar plates.

In eight out of 18 attempts at isolation from the osteomyelitis lesions a pure growth was obtained. Subsequent tests indicated that all eight were identical. In the other ten cases no bacteria could be grown. The organism was a small, Gram-negative motile coccobacillus. Growth occurred within 24 hours' incubation at $37^{\circ} \mathrm{C}$ and at room temperature. The organism grew on blood agar, and on MacConkey and plain agar both aerobically and anaerobically. The colonies were small, white or translucent, non-lactose fermenting and non-haemolytic. In plain broth at $37^{\circ} \mathrm{C}$ growth occurred in floccules. The following biochemical behaviour was observed. Glucose, mannitol and glycerol were fermented with the formation of acid but no gas. Lactose, sucrose and dulcitol were not fermented. Indole and $\mathrm{H}_{2} \mathrm{~S}$ were not

TABLE

The effect on 9-wk-old turkeys of intravenous injection of Yersinia pseudotuberculosis

\begin{tabular}{|c|c|c|c|c|c|c|}
\hline \multirow{2}{*}{ Number of } & \multicolumn{5}{|c|}{ Number of organisms injected } & \multirow{2}{*}{$\begin{array}{l}\text { Total number } \\
\text { of turkeys }\end{array}$} \\
\hline & $3.2 \times 10^{6}$ & $6.4 \times 10^{5}$ & $3.2 \times 10^{5}$ & $3 \cdot 2 \times 10^{4}$ & $3 \cdot 2 \times 10^{3}$ & \\
\hline $\begin{array}{l}\text { Turkeys used } \\
\text { Deaths } \\
\text { Birds with granulomatous } \\
\text { lesions in viscera }\end{array}$ & $\begin{array}{l}6 \\
2 \\
3^{*}\end{array}$ & $\begin{array}{l}6 \\
0 \\
2\end{array}$ & $\begin{array}{l}6 \\
0 \\
0\end{array}$ & $\begin{array}{l}6 \\
0 \\
0\end{array}$ & $\begin{array}{l}4 \\
0 \\
0\end{array}$ & $\begin{array}{r}28 \\
2 \\
5\end{array}$ \\
\hline $\begin{array}{l}\text { Lame birds } \\
\text { Birds with osteomyelitis }\end{array}$ & $\begin{array}{l}4 \\
5 \dagger\end{array}$ & $\begin{array}{l}5 \\
5\end{array}$ & $\begin{array}{l}5 \\
6\end{array}$ & $\begin{array}{l}2 \\
3\end{array}$ & $\begin{array}{l}2 \\
4\end{array}$ & $\begin{array}{l}18 \\
23\end{array}$ \\
\hline
\end{tabular}

* Including two birds that died of the infection.

$\dagger$ Including one bird that died of the infection.

produced. Urea was broken down to ammonia, and the nitrate reduction and methyl red tests were positive. The Voges-Proskauer and Koser's citrate utilisation tests were negative. These findings suggested that the organism was $Y$. pseudotuberculosis. Dr N. S. Mair of the Public Health Laboratory, Leicester, confirmed the identification and classified the organism as belonging to serotype IA.

Pathogenicity of the organism. Two $\mathrm{ml}$ of an 18-hr broth culture of $Y$. pseudotuberculosis incubated at $30^{\circ} \mathrm{C}$ were inoculated intravenously into each of three 8-wk-old chickens and subcutaneously into each of two adult male guinea-pigs; $0.5 \mathrm{ml}$ of the same culture was inoculated intraperitoneally into each of six white mice.

The chickens and mice all died within $48 \mathrm{hr}$ and the organism was recovered from the heart blood, liver, kidney and spleen in all cases. No macroscopic lesions were visible at necropsy in any of the animals.

The guinea-pigs became sick within $24 \mathrm{hr}$ and showed signs of inappetence and dyspnoea. They were killed after 5 days; necropsy revealed caseous, indurated lesions at the inoculation sites. The liver and spleen contained numerous small, white, granulomatous lesions from which the organisms could be recovered. No Y. pseudotuberculosis could be cultured from the heart blood.

Experimental transmission in turkeys. Thirty-two 9-wk-old turkeys were bought from a turkey farm for use in the transmission studies. Four of them were housed separately as controls to the experimental group of 28 .

An 18-hr broth culture grown at $30^{\circ} \mathrm{C}$ was used. A viable count was made and simultaneously various dilutions were inoculated intravenously into groups of turkeys. After infection, all birds were observed daily. One bird in the group receiving the highest inoculum died on the 5th day and another on the 10th day after infection. None 
of the others died. Lameness was first noticed 6 days after infection and approximately half of the birds showed signs of locomotor difficulty by the 10th day. The 26 turkeys surviving infection were killed at intervals from day 11 to day 31 . The control group of four uninoculated turkeys, which remained clinically normal throughout the course of the experiment, were killed at the same time as the last birds of the infected group.

All the turkeys were examined at necropsy and attempts were made to recover the organism from the livers and osteomyelitic lesions of some of the birds.

The results of infection with the various dosages are summarised in the table. No lesions were found in any of the control turkeys. Although two turkeys died with classical, chronic pseudotuberculosis and three others showed typical granulomatous lesions of the liver and spleen, the majority of birds were like those suffering from the natural condition and their bone lesions were identical in appearance. These were found at both ends of all thelong bones of the legs and wings, although not all bones were affected in every bird. Y. pseudotuberculosis was recovered from the livers in which granulomatous lesions were found, but not from other livers. The organism could also be recovered from a proportion of osteomyelitic lesions-even from those cultured 31 days after infection of the birds. In five cases the joint cavities (all stifle joints) showed excessive fluid, which was thick, grey and viscous. The organism could not be recovered from this exudate.

\section{Discussion}

The outbreak of pseudotuberculosis described in this paper appears to be markedly different in its manifestations from all previously reported cases. The organism isolated, however, belonged to the commonest serotype of Y. pseudotuberculosis, type IA, and none of its biochemical properties that were tested were in any way atypical.

Locomotor difficulties, particularly in heavily built strains, are becoming increasingly common in turkeys. The clinical and pathological findings in affected turkeys described in this paper are identical with those described by Nairn (1969). It is possible that the cartilage growth plates of the long bones of modern breeds of turkey are, for some unknown reason, more susceptible to bacterial invasion than were the growth plates of their more slowly growing forebears.

\section{SUMMARY}

An outbreak of lameness with sudden onset and high morbidity in a flock of 12-wk-old turkeys is reported. The lameness was associated with osteomyelitis caused by Yersinia pseudotuberculosis serotype IA. The condition was reproduced experimentally.

We are grateful to Dr N. S. Mair for his advice and for the serological typing of the organisms.

\section{REFERENCES}

Blaxland, J. D. 1947. Pasteurella pseudotuberculosis infection in turkeys. Vet. Rec., $59,317$.

Carnaghan, R. B. A. 1966. Spinal cord compression in fowls due to spondylitis caused by Staphylococcus pyogenes. J. Comp. Path., 76, 9.

NAIRN, M. E. 1969. Osteomyelitis-a common cause of lameness in the growing turkey. Nicholas Turkey News, 12, no. 11.

Snoeyendos, G. H. 1965. Pseudotuberculosis. In Diseases of poultry, 5th ed., edited by H. E. Biester and L. H. Schwarte, Ames, Iowa, p. 435. 